\title{
Dense Colloidal Suspensions under Time-Dependent Shear
}

\author{
J. M. Brader, ${ }^{1}$ Th. Voigtmann, ${ }^{2}$ M. E. Cates,${ }^{2}$ and M. Fuchs ${ }^{1}$ \\ ${ }^{1}$ Fachbereich Physik, Universität Konstanz, D-78457 Konstanz, Germany \\ ${ }^{2}$ SUPA, School of Physics, The University of Edinburgh, Mayfield Road, Edinburgh EH9 3JZ, United Kingdom
}

(Received 24 August 2006; published 31 January 2007)

\begin{abstract}
We consider the nonlinear rheology of dense colloidal suspensions under a time-dependent simple shear flow. Starting from the Smoluchowski equation for interacting Brownian particles advected by shearing (ignoring fluctuations in fluid velocity), we develop a formalism which enables the calculation of timedependent, far-from-equilibrium averages. Taking shear stress as an example, we derive exactly a generalized Green-Kubo relation and an equation of motion for the transient density correlator, involving a three-time memory function. Mode coupling approximations give a closed constitutive equation yielding the time-dependent stress for arbitrary shear rate history. We solve this equation numerically for the special case of a hard sphere glass subject to step strain.
\end{abstract}

PACS numbers: 82.70.Dd, 64.70.Pf, 83.10.Gr, 83.60.Df

The constitutive equations of a material relate its stress tensor $\boldsymbol{\sigma}(t)$ at time $t$ to its flow history, described by a strain-rate tensor $\boldsymbol{\kappa}\left(t^{\prime}<t\right)$. Dense suspensions of colloidal particles, close to the glass transition, show strongly viscoelastic behavior and nonlinear shear response; their constitutive equations must be highly nontrivial [1]. In this Letter, we report first-principles work aimed at elucidating these equations theoretically.

Alongside phenomenological treatments (e.g., [2]), several approaches to the rheology of glassy materials have been proposed. The mesoscopic soft glassy rheology (SGR) model [3] mimics the glass transition by a oneparticle hopping dynamics in a well chosen distribution of trap depths [4]. While the predictions of SGR are broadly consistent with experiments on many soft materials [5], it does not capture the discontinuous jump in yield stress on glass formation observed in experiments on colloidal suspensions of hard spheres [1]. The same is true of spin glass approaches which describe a different phenomenology akin to "power-law yielding materials" [6]. The observed yield stress is captured, however, by a first-principles approach to colloid rheology, based on mode coupling theory (MCT), which has recently been formulated for systems under steady shear [7]. The MCT has had considerable semiquantitative success in accounting for the interaction dependence of the static glass transition $[8,9]$ and the time dependence of light scattering correlators [10] from microscopic starting points. The MCT also gives sensible predictions for the viscoelastic spectrum $G^{*}(\omega)$ as measured in linear rheology $[11,12]$.

In this Letter, we develop a first-principles description of the far-from-equilibrium states of dense colloids under prescribed time-dependent shear flow. For a system of interacting particles advected by the imposed strain rate $\boldsymbol{\kappa}$, we begin by developing a formalism which facilitates the calculation of general time-dependent averages. Using this formalism, we derive formally exact generalized Green-Kubo equations, taking the shear stress as an example. These expressions can be approximated in terms of transient two-time density correlators. We find an exact equation of motion for the correlator which, for the general strain rate, displays a remarkable three-time structure to the memory function. Finally, we make an MCT-based closure of this equation. To show that the theory yields sensible predictions for strongly time-dependent flows, we consider step strain as a specific example. Our approach is valid for all homogeneous and incompressible flows which satisfy $\boldsymbol{\kappa}(t) \cdot \boldsymbol{\kappa}(t)=0$. For clarity of presentation, we focus on the case of simple shearing with fixed axes (velocity along $x$, gradient along $y$ ) and an arbitrary time-dependent strain rate $\dot{\gamma}(t)$, so that $\boldsymbol{\kappa}_{i j}=\dot{\gamma}(t) \delta_{i x} \delta_{j y}$.

Our findings highlight the formal importance of "integration through transients" (ITT) [7] in preparing the best ground for judicious application of the MCT. Indeed, a somewhat simpler MCT-inspired approach to colloid rheology was developed in Refs. $[13,14]$ (an alternative simplified MCT approach is presented in Ref. [15]), which for fluid states in steady shear gives broadly similar results to those of Ref. [7]. However, its recent extension to a specific time-dependent flow (oscillatory) [14] gives equations structured quite differently from ours; in particular, there is no sign of a third time in the memory function, which we show to be an exact consequence of the Smoluchowski equation and which is preserved in our approximations.

We start with a system of $N$ spherical Brownian particles of diameter $d$ interacting via internal forces $\mathbf{F}_{i}, i=$ $1, \cdots, N$, and dispersed in a solvent with a specified time-dependent velocity profile $\mathbf{v}(\mathbf{r}, t)=\boldsymbol{\kappa}(t) \cdot \mathbf{r}$. This neglects velocity fluctuations and, hence, hydrodynamic interactions [7]. This is defensible for systems close to the glass transition and at low shear rates, where particle motions are dominated by excluded volume constraints and individual lubrication contacts are short-lived on the time scale for structural rearrangement; however, it may fail as random close packing is approached and/or at higher shear rates, where, e.g., shear thickening is seen [16]. The distribution function of particle positions evolves accord- 
ing to the Smoluchowski equation $[17,18]$

$$
\partial_{t} \Psi(t)=\Omega(t) \Psi(t), \quad \Omega(t) \equiv \sum_{i} \boldsymbol{\partial}_{i} \cdot\left[\boldsymbol{\partial}_{i}-\mathbf{F}_{i}-\boldsymbol{\kappa}(t) \cdot \mathbf{r}_{i}\right],
$$

where $\Omega(t)$ is the Smoluchowski operator. We choose length, energy, and time units such that $d=k_{B} T=D_{0}=$ 1. Translational invariance of the sheared system leads to a coupling between a density fluctuation with preadvected wave vector $\mathbf{q}\left(t, t^{\prime}\right)=\mathbf{q}-\mathbf{q} \cdot \int_{t^{\prime}}^{t} d s \boldsymbol{\kappa}(s)$ at time $t^{\prime}$ and another with wave vector $\mathbf{q}$ at later time $t$. Wave vector advection is how strain enters our formalism and accounts for the affine part of the particle motion.

To find the distribution function $\Psi(t)$, we start from a system in quiescent equilibrium in the infinite past and obtain $\Psi(t)$ by following the complete shear history. This explicitly excludes aging effects arising from nonergodicity in the initial state. Integrating the Smoluchowski equation, we obtain the formal expression [19]

$$
\Psi(t)=\Psi_{e}+\int_{-\infty}^{t} d t^{\prime} \Psi_{e} \operatorname{Tr}\left\{\boldsymbol{\kappa}\left(t^{\prime}\right) \hat{\boldsymbol{\sigma}}\right\} e^{\int_{t^{\prime}}^{t} d s \Omega^{\dagger}(s)},
$$

where $\hat{\boldsymbol{\sigma}}$ is the zero wave vector limit of the potential part of the stress tensor and $\Psi_{e}$ is the equilibrium distribution function. The adjoint Smoluchowski operator can be obtained by partial integration and is given by $\Omega^{\dagger}(t)=$ $\sum_{i}\left[\boldsymbol{\partial}_{i}+\mathbf{F}_{i}+\mathbf{r}_{i} \cdot \boldsymbol{\kappa}^{T}(t)\right] \cdot \boldsymbol{\partial}_{i}$. The time ordered exponential $e_{-}$imposes that later times appear on the right and arises because the operator $\Omega(t)$ does not commute with itself at different times. Equation (1) generalizes the ITT method developed in Ref. [7] and is to be used with the understanding that functions to be averaged are placed to the right of the operators and then integrated over particle coordinates. A general function $f$ of the phase space coordinates thus has the time-dependent average $\langle f\rangle(t)=$ $\langle f\rangle+\int_{-\infty}^{t} d t^{\prime}\left\langle\operatorname{Tr}\left\{\boldsymbol{\kappa}\left(t^{\prime}\right) \hat{\boldsymbol{\sigma}}\right\} \exp _{-}\left[\int_{t^{\prime}}^{t} d s \Omega^{\dagger}(s)\right] f\right\rangle$ with respect to the distribution function $(1)$, where $\langle\cdot\rangle$ indicates averaging with respect to the equilibrium distribution. By choosing $f=\sigma_{x y} / V$ [20], we obtain an exact generalized Green-Kubo relation for the time-dependent shear stress (in volume $V$ )

$$
\sigma(t)=\int_{-\infty}^{t} d t^{\prime} \dot{\gamma}\left(t^{\prime}\right)\left[\frac{1}{V}\left\langle\sigma_{x y} e^{\int_{t^{\prime}}^{t} d s \Omega^{\dagger}(s)} \sigma_{x y}\right\rangle\right],
$$

where the factor $[\cdot]$ can be identified formally as $G\left(t, t^{\prime},[\dot{\gamma}]\right)$, a time-dependent shear modulus. Replacing $\Omega^{\dagger}(t)$ with the quiescent-state operator recovers the linear response. Equation (2) opens a route to calculate $\sigma(t)$ for a given flow history $\dot{\gamma}(t)$. The ITT method based on Eq. (1) also yields correlators, distorted structure factors, and susceptibilities, to be detailed elsewhere.

To approximate our formally exact result (2), we now project onto densities $\rho_{\mathbf{q}}=\sum_{i} e^{i \mathbf{q} \cdot \mathbf{r}_{i}}$ and density pairs (given by the square of the density in real space) $[7,8]$. This physical approximation amounts to assuming that these are the only slow variables, sufficient to describe the relaxation of the local structure in the glassy regime.
The resulting shear stress is given by

$$
\sigma(t)=\int_{-\infty}^{t} d t^{\prime} \dot{\gamma}\left(t^{\prime}\right) \int \frac{d \mathbf{k}}{16 \pi^{3}} \frac{k_{x}^{2} k_{y} k_{y}\left(t, t^{\prime}\right)}{k k\left(t, t^{\prime}\right)} \frac{S_{k}^{\prime} S_{k\left(t, t^{\prime}\right)}^{\prime}}{S_{k\left(t, t^{\prime}\right)}^{2}} \Phi_{\mathbf{k}}^{2}\left(t, t^{\prime}\right),
$$

where $\Phi_{\mathbf{k}}\left(t, t^{\prime}\right)$ is the transient density correlator, which (in the absence of time translational invariance) is a function of two times, and $S_{k}^{\prime}=d S_{k} / d k$, with $S_{k}$ the equilibrium static structure factor. The projection onto density pairs means that the interparticle forces $\mathbf{F}_{i}$ are fully determined from $S_{k}$ and density fluctuations. This MCT-based approximation is well-tested, although if the equal-time structure under shear deviates strongly enough from $S_{k}$ to enter an anharmonic regime, improvements to it may be needed [7,21]. In Eq. (3), the term $\Phi_{\mathbf{k}}^{2}\left(t, t^{\prime}\right)$ can be viewed as the "survival probability" to time $t$ of a stress contribution created by an initial step strain applied at earlier time $t^{\prime}$; the remaining factor is the stress per unit initial strain. The transient density correlator required in Eq. (3) is defined as $\Phi_{\mathbf{q}}\left(t, t^{\prime}\right)=\left\langle\rho_{\mathbf{q}}^{*} \exp _{-}\left[\int_{t^{\prime}}^{t} d s \Omega^{\dagger}(s)\right] \rho_{\mathbf{q}\left(t, t^{\prime}\right)}\right\rangle /\left(N S_{q}\right)$ and is a key quantity within our approach. Note that it contains only information on the strain accumulated between the two correlated times $t^{\prime}$ and $t$ and is independent of the strain history for times earlier than $t^{\prime}$.

Equation (3) gives the stress in terms of $\Phi_{\mathbf{k}}\left(t, t^{\prime}\right)$, which is itself dependent on flow history. Using Zwanzig-Moritype projection operator manipulations and applying the theory of Volterra integral equations, we obtain the following formally exact results:

$$
\begin{aligned}
\frac{\partial}{\partial t} \Phi_{\mathbf{q}}\left(t, t_{0}\right)+ & \Gamma_{\mathbf{q}}\left(t, t_{0}\right)\left(\Phi_{\mathbf{q}}\left(t, t_{0}\right)\right. \\
& \left.+\int_{t_{0}}^{t} d t^{\prime} m_{\mathbf{q}}\left(t, t^{\prime}, t_{0}\right) \frac{\partial}{\partial t^{\prime}} \Phi_{\mathbf{q}}\left(t^{\prime}, t_{0}\right)\right)=0, \\
\Gamma_{\mathbf{q}}\left(t, t_{0}\right)= & -\frac{1}{S_{\mathbf{q}}}\left\langle\rho_{\mathbf{q}}^{*} \Omega_{s}\left(t, t_{0}\right) \rho_{\mathbf{q}}^{s}\right\rangle \\
= & \frac{q^{2}}{S_{q}}-\mathbf{q} \cdot \boldsymbol{\kappa}_{t t_{0}} \cdot \mathbf{q} \frac{1+S_{q}}{S_{q}}+\left|\mathbf{q} \cdot \boldsymbol{\kappa}_{t t_{0}}\right|^{2}, \\
m_{\mathbf{q}}\left(t, t^{\prime}, t_{0}\right)= & \frac{-\left\langle\rho_{\mathbf{q}}^{*} \Omega_{s}\left(t^{\prime}, t_{0}\right) U\left(t, t^{\prime}, t_{0}\right) Q_{s} \Omega_{s}\left(t, t_{0}\right) \rho_{\mathbf{q}}^{s}\right\rangle}{S_{q} \Gamma_{\mathbf{q}}\left(t^{\prime}, t_{0}\right) \Gamma_{\mathbf{q}}\left(t, t_{0}\right)} .
\end{aligned}
$$

In the equation of motion (4) for $\Phi_{\mathbf{k}}\left(t, t^{\prime}\right)$, the initial decay rate $\Gamma_{\mathbf{q}}\left(t, t^{\prime}\right)$ describes Taylor dispersion, which enhances diffusion in the direction of flow [17]. For our chosen flow geometry, this can be calculated explicitly as given in Eq. (5), where $\boldsymbol{\kappa}_{t t_{0}}=\int_{t_{0}}^{t} d s \boldsymbol{\kappa}(s)$ is the shear strain accumulated between $t_{0}$ and $t$. The memory function $m_{\mathbf{q}}\left(t, t^{\prime}, t_{0}\right)$ in Eq. (4) describes competition between shearing and the cage effect responsible for slow structural relaxation; strikingly, this is a function of three times, not two. It is useful to interpret $m_{\mathbf{q}}\left(t, t^{\prime}, t_{0}\right)$ as describing the decay of memory between times $t^{\prime}$ and $t$, in the presence of shear, allowing for the coupling to stress in the system that is still relaxing 
from the strain accumulated between $t_{0}$ and $t^{\prime}$. The time $t_{0}$ enters the theory in a parametric fashion and is quite distinct in character from the two later times. Equation (6) for $m_{\mathbf{q}}$ involves the propagator $U\left(t, t^{\prime}, t_{0}\right)=$ $\exp _{-}\left[\int_{t^{\prime}}^{t} d s^{\prime} Q_{s} \Omega_{s}^{\mathrm{irr}}\left(s^{\prime}, t_{0}\right)\right]$, where $\Omega_{s}^{\mathrm{irr}}\left(t, t^{\prime}\right)$ is the singleparticle-irreducible operator [7,22] and $Q_{s}$ is an equilibrium projector orthogonal to density fluctuations. In deriving these formal results, we introduced $\rho_{\mathbf{q}}^{s}=e^{i \mathbf{q} \cdot \mathbf{r}_{s}}$, the density of a single tagged particle, whose motion is described by $\Omega_{s}\left(t, t_{0}\right)=-i \mathbf{q} \cdot \boldsymbol{\kappa}(t) \cdot \mathbf{r}_{s}-i \mathbf{q} \cdot \boldsymbol{\kappa}_{t t_{0}} \cdot\left(2 \boldsymbol{\partial}_{s}+\right.$ $\left.\mathbf{F}_{s}\right)+\Omega^{\dagger}(t)-\mathbf{q} \cdot \boldsymbol{\kappa}_{t t_{0}} \cdot \boldsymbol{\kappa}_{t t_{0}}^{T} \cdot \mathbf{q}$.

To close Eqs. (4)-(6) for the transient density correlator, we now make an MCT-based approximation to the average in Eq. (6). Taking care to preserve the translational invariance of $m_{\mathbf{q}}\left(t, t^{\prime}, t_{0}\right)$, we obtain

$$
\begin{aligned}
m_{\mathbf{q}}\left(t, t^{\prime}, t_{0}\right)= & \frac{\rho}{16 \pi^{3}} \int d \mathbf{k} \frac{S_{k} S_{p} V_{\mathbf{q} \mathbf{k p}}^{(1)} V_{\mathbf{q} \mathbf{k p}}^{(2)} \Phi_{\mathbf{k}}\left(t, t^{\prime}\right) \Phi_{\mathbf{p}}\left(t, t^{\prime}\right)}{S_{q} \Gamma_{\mathbf{q}}\left(t^{\prime}, t_{0}\right) \Gamma_{\mathbf{q}}\left(t, t_{0}\right)}, \\
V_{\mathbf{q} \mathbf{k p}}^{(1)}= & \tilde{\mathbf{q}} \cdot\left[\hat{\mathbf{k}} c_{\hat{k}}+\hat{\mathbf{p}} c_{\hat{p}}+S_{q}\left(\hat{\mathbf{q}} c_{\hat{q}}-\mathbf{q} c_{q}\right)\right], \\
V_{\mathbf{q} \mathbf{k}}^{(2)}= & \mathbf{q} \cdot\left[\mathbf{k} c_{k}+\mathbf{p} c_{p}+\boldsymbol{\kappa}_{t^{\prime} t_{0}} \cdot \mathbf{q} S_{q}\left\{c_{k}+c_{p}\right.\right. \\
& \left.\left.-\frac{\rho}{2}\left(c_{q} c_{k}+c_{q} c_{p}+c_{k} c_{p}\right)\right\}\right],
\end{aligned}
$$

where $\mathbf{p}=\mathbf{q}-\mathbf{k} ; \boldsymbol{\rho}=N / V ; \boldsymbol{\rho} c_{q}=1-1 / S_{q}$. The wave vectors $\tilde{\mathbf{q}}=\mathbf{q}-\mathbf{q} \cdot \boldsymbol{\kappa}_{t t_{0}}$ and $\hat{\mathbf{q}}=\mathbf{q}-\mathbf{q} \cdot \boldsymbol{\kappa}_{t t^{\prime}}$ contain shear strains accumulated over different temporal ranges.

Equations (3) - (5) and (7) form a closed set of equations to predict the shear stress for arbitrary time-dependent shear flows of the form $\boldsymbol{\kappa}=\dot{\gamma}(t) \delta_{i x} \delta_{j y}$. Other than $\dot{\gamma}(t)$, the only required inputs are the density $\rho$ and the static structure factor $S_{k}$ in the unsheared state. The parametric nature of $t_{0}$ is made explicit in the MCT approximation (7), where all three times enter the vertex functions $V^{(1,2)}$, but only $t^{\prime}$ and $t$ enter the correlators. For $\dot{\gamma}=0$, our equations reduce to those of quiescent MCT [11] and for steady shear to those of Ref. [7].

In developing nonlinear constitutive equations, it is helpful to study nonlinear step strain as a benchmark. In an idealized step-strain experiment, the shear rate is given by $\dot{\gamma}(t)=\gamma \delta\left(t-t_{0}\right)$, which provides a demanding test of any constitutive equation. For step strain, our approximate Green-Kubo relation (3) reduces to

$$
\sigma(t)=\int d \mathbf{k}\left\{\frac{\gamma}{16 \pi^{3}} \frac{k_{x}^{2} k_{y} k_{y}^{\gamma}}{k k^{\gamma}} \frac{S_{k}^{\prime} S_{k^{\gamma}}^{\prime}}{S_{k^{\gamma}}^{2}}\right\}\left[\Phi_{\mathbf{k}}^{\gamma}\left(t, t_{0}\right)\right]^{2},
$$

where $t>t_{0}$ and $\mathbf{k}^{\gamma}=\left(k_{x}, k_{y}-\frac{1}{2} \gamma k_{x}, k_{z}\right)$. We have included an additional superscript on the correlator to make explicit the strain dependence. The initial decay rate becomes independent of time, $\Gamma_{\mathbf{q}}^{\gamma}=q^{2} / S_{q}+q_{x} q_{y} \gamma\left(1+S_{q}\right) /\left(2 S_{q}\right)+$ $q_{x}^{2} \gamma^{2} / 4$, as the time $t_{0}$ drops out in favor of an explicit $\gamma$ dependence. A similar reduction occurs for the memory function, leading us to modify the notation $m_{\mathbf{q}}\left(t, t^{\prime}, t_{0}\right) \rightarrow$ $m_{\mathbf{q}}^{\gamma}\left(t, t^{\prime}\right)$. The memory function (7) becomes $m_{\mathbf{q}}^{\gamma}\left(t, t^{\prime}\right)=\frac{\rho}{16 \pi^{3}} \int d \mathbf{k} \frac{S_{k} S_{p} V_{\mathbf{q} \mathbf{k}}^{(1)} V_{\mathbf{q} \mathbf{k}}^{(2)} \Phi_{\mathbf{k}}^{\gamma=0}\left(t, t^{\prime}\right) \Phi_{\mathbf{p}}^{\gamma=0}\left(t, t^{\prime}\right)}{S_{q} \Gamma_{\mathbf{q}}^{\gamma} \Gamma_{\mathbf{q}}^{\gamma}}$,

with $\tilde{\mathbf{q}} \rightarrow \mathbf{q}^{\gamma}$, hatted variables made unhatted, and $\boldsymbol{\kappa}_{t t_{0}} \rightarrow$ $\kappa_{i j}^{\gamma}=\frac{1}{2} \gamma \delta_{i x} \delta_{j y}$ in the vertex expressions of Eq. (7). The strain is zero in the two correlators since there is no strain imposed between $t^{\prime}$ and $t$; these are quiescent MCT correlators. Thus, all of the $\gamma$ dependence in the memory function stems from the presence of $t_{0}$ in Eq. (7). In Eq. (9), the forces represented by the vertices are straindependent but relaxation of the structure is $\gamma$-independent. We thus obtain a simplified equation of motion for the correlators needed in (8). At state points for which quiescent MCT predicts a glass, the memory function remains nonergodic for all values of $\gamma$. The glasses do not become fluidized permanently under step strain. The equation of motion for $\Phi_{\mathbf{q}}^{\gamma}\left(t, t_{0}\right)$ is a linear equation in $\Phi_{\mathbf{q}}^{\gamma}\left(t, t_{0}\right)$ with given non-Markovian memory function. Because of the zero duration of the applied strain, we can make the replacements $\Phi_{\mathbf{q}}^{\gamma}\left(t, t_{0}\right) \rightarrow \Phi_{\mathbf{q}}^{\gamma}\left(t-t_{0}\right)$ and $m_{\mathbf{q}}^{\gamma}\left(t, t^{\prime}\right) \rightarrow m_{\mathbf{q}}^{\gamma}(t-$ $\left.t^{\prime}\right)$ without further approximation.

In practice, the presence of anisotropy still poses difficulties for numerical computation; specializing to the case of hard spheres, we have therefore solved Eq. (8) for the shear stress $\sigma(t)$ within the isotropic approximation $[7,14,23]$. The $S_{k}$ input is taken from the Percus-Yevick theory, which yields a glass transition at $\phi_{\mathrm{MCT}} \equiv$ $\pi d^{3} \rho / 6=0.516$ with our present numerical discretization [24]. In Fig. 1, we show the long-time stress $\sigma(\infty)$ as a function of strain amplitude $\gamma$ for a state point just above the MCT glass transition $[\phi=0.52$; for fluid states, we of course find $\sigma(\infty)=0$ ]. As $\gamma$ is increased, we first obtain the expected linear response behavior before entering a regime of sublinear increase, indicating the onset of plastic flow. (A regime of linear response persists even at the critical point $\phi=\phi_{c}$.) For large $\gamma$ values, our calculations

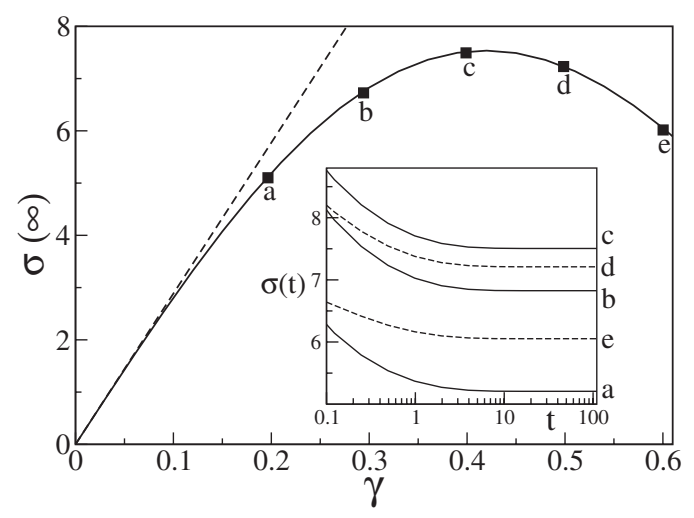

FIG. 1. The long-time stress $\sigma(\infty)$ as a function of strain $\gamma$ for a hard sphere glass just above the MCT glass transition $(\phi=$ $0.52)$ following a step strain. The dashed line is the linear response result, and the solid line is $\sigma(\infty)$ from the isotropic approximation [23]. The inset shows the decaying stress as a function of time following the step. 
yield unphysical negative $\sigma(\infty)$. This may reflect a shortcoming of MCT, where for large $\gamma$ use of $S_{k}$ to proxy the colloidal interactions becomes questionable. The inset shows $\sigma(t)$ for various values of $\gamma$. In contrast to polymer melts [18] and some reports on colloidal fluids [25], $\sigma(t)$ for the present hard sphere glass is generally not strainfactorable. The results (Fig. 1) are in qualitative agreement with recent step-strain experiments on suspensions of hardsphere particles above the glass transition [26]. The experimental data show a peak and a region of negative slope in $\sigma(\infty)$ in accord with our findings [27]. The isotropic approximation is known to underestimate the effects of shearing [7]; a fuller treatment might shift the peak in $\sigma(\infty)$ from $\gamma \simeq 0.4$ closer to the experimental results [26], which peak at $\gamma \simeq 0.1$. Note also that in the experiments the strain is ramped up over some short finite interval, during which additional plastic rearrangement may occur. Our general expressions (3)-(5) and (7) should capture this, although (8) and (9) clearly do not.

To summarize, for interacting Brownian particles advected by a nonfluctuating shear flow, we have generalized the integration through transients formalism of Ref. [7] to address arbitrary far-from-equilibrium, time-dependent shearing. When complemented with mode-coupling approximations, this provides a route to calculating timedependent averages in the sheared system. To demonstrate this approach, we have presented a Green-Kubo-type relation for the shear stress and an equation of motion for the transient density correlator. MCT closure of these expressions yielded a first-principles constitutive model for the shear rheology of dense suspensions close to the glass transition. In step strain, this predicts first a linear regime followed by plastic deformation of the glass with a maximum in the long-time stress, as seen experimentally.

We neglected extensional flow, and we assumed a spatially homogeneous strain rate $\dot{\gamma}(t)$. Nonetheless, the work goes far beyond linear response [12]: Applying the theory to the case of oscillatory shear would predict straindependent storage and loss moduli including "higher harmonic" contributions [1,28]. Efficient numerical algorithms to tackle the anisotropy and loss of time translation invariance are under development. The developments presented here form a secure starting point both for more complete theories, in which the MCT assumptions might be partially relaxed (for instance, to address aging behavior), and for schematic models that simplify the algebra but add extra physics such as anharmonicity, shear thickening, and jamming [21].

We acknowledge the Transregio SFB TR6, No. EPSRC/ GR/S10377, and DFG No. Vo 1270/1-1 for financial support. We thank Stefan Egelhaaf, Marco Laurati, and Martin Greenall for helpful discussions.

[1] G. Petekidis, D. Vlassopoulos, and P. N. Pusey, Faraday Discuss. 123, 287 (2003); J. Phys.
Condens. Matter 16, S3955 (2004), and references therein.

[2] C. Derec, A. Ajdari, and F. Lequeux, Eur. Phys. J. E 4, 355 (2001).

[3] P. Sollich et al., Phys. Rev. Lett. 78, 2020 (1997); S. Fielding, P. Sollich, and M.E. Cates, J. Rheol. (N.Y.) 44, 323 (2000); M. E. Cates and P. Sollich, J. Rheol. (N.Y.) 48, 193 (2004).

[4] J.-P. Bouchaud, J. Phys. I (France) 2, 1705 (1992).

[5] S. D. Holdsworth, Chem. Eng. Res. Des. 71, 139 (1993).

[6] L. Berthier et al., Phys. Rev. E 61, 5464 (2000).

[7] M. Fuchs and M.E. Cates, Phys. Rev. Lett. 89, 248304 (2002); J. Phys. Condens. Matter 17, S1681 (2005); M. E. Cates et al., in Unifying Concepts in Granular Media and Glasses, edited by A. Coniglio et al. (Elsevier, Amsterdam, 2004), p. 203.

[8] W. Götze and L. Sjoegren, Rep. Prog. Phys. 55, 241 (1992).

[9] K. N. Pham et al., Science 296, 104 (2002).

[10] W. van Megen and S. M. Underwood, Phys. Rev. Lett. 70, 2766 (1993); Phys. Rev. E 49, 4206 (1994); T. Eckert and E. Bartsch, Faraday Discuss. 123, 51 (2003).

[11] W. Götze, in Liquids, Freezing and Glass Transition, edited by J-P. Hansen, D. Levesque, and J. Zinn-Justin (North-Holland, Amsterdam, 1991), p. 287.

[12] G. Nägele and J. Bergenholtz, J. Chem. Phys. 108, 9893 (1998); M. Fuchs and M. R. Mayr, Phys. Rev. E 60, 5742 (1999).

[13] K. Miyazaki and D. R. Reichman, Phys. Rev. E 66, 050501(R) (2002); K. Miyazaki, D. R. Reichman, and R. Yamamoto, Phys. Rev. E 70, 011501 (2004).

[14] K. Miyazaki et al., Europhys. Lett. 75, 915 (2006).

[15] V. Kobelev and K. S. Schweizer, Phys. Rev. E 71, 021401 (2005).

[16] J. W. Bender and N. J. Wagner, J. Rheol. (N.Y.) 40, 899 (1996).

[17] J.K. G. Dhont, An Introduction to the Dynamics of Colloids (Elsevier, Amsterdam, 1996).

[18] M. Doi and S.F. Edwards, The Theory of Polymer Dynamics (Oxford University, New York, 1989).

[19] In cases where phase space decomposes into disjoint pockets ("nonmixing dynamics"), the distribution function of Eq. (2) averages over all compartments.

[20] Normal stresses can be found the same way by choosing $f=\left(\sigma_{x x}-\sigma_{y y}\right) / V$, etc., and deriving analogs to Eq. (3).

[21] C. B. Holmes et al., J. Rheol. (N.Y.) 49, 237 (2005).

[22] B. Cichocki and W. Hess, Physica (Amsterdam) 141A, 475 (1987).

[23] In the isotropic approximation, we replace both the numerator and the denominator in (9) by the angle average over $\mathbf{q}$ to obtain an isotropic memory function $m_{q}^{\gamma}\left(t, t^{\prime}\right)$. If we also isotropize the initial decay rate, then we obtain an isotropic correlator. This is then used in Eq. (8).

[24] T. Franosch et al. Phys. Rev. E 55, 7153 (1997).

[25] H. Watanabe et al. Rheol. Acta 35, 433 (1996).

[26] K. N. Pham et al. Europhys. Lett. 75, 624 (2006); W. C. K. Poon (private communication).

[27] Note that $d \sigma / d \gamma<0$ for an elastic system would cause a mechanical (static shear-banding) instability. However, since $\gamma$ denotes an initial strain and $\sigma(\infty)$ a final stress, this might not arise under plastic flow conditions. No shear banding is reported in Ref. [26].

[28] M. Wilhelm, Macromol. Mater. Eng. 287, 83 (2002). 